\title{
Le Groupe de Liège autour de Jacques Izoard
}

\section{Gérald Purnelle}

L'existence d'un «Groupe de Liège » autour du poète Jacques Izoard a déjà été abordée à deux reprises, dans des études plus générales ${ }^{1}$. Il s'agira ici de reprendre le sujet pour luimême, en partant de documents d'époque, afin d'observer la façon dont l'existence dudit groupe s'est affirmée au milieu des années 1970.

\section{CONTEXTE}

\section{LE CHAMP LIÉGEOIS}

Dans l'histoire de la ville et de la région de Liège, les années 1970 sont marquées par un vaste mouvement d'ouverture vers la modernité dans les domaines sociaux, intellectuels, culturels et artistiques $^{2}$, dans une ville à vocation de métropole locale qui s'était jusqu'alors généralement tenue à l'écart des avant-gardes et de la modernité culturelle. Auparavant, au plan littéraire, Denis et Klinkenberg relèvent en effet qu'il y eut toujours à Liège une « relative timidité en matière esthétique, caractérisée soit par le compromis moderniste entre tradition et modernité, soit par un repli caractérisé sur l'esthétique classique, au nom d'une sensibilité qui a parfois été décrite comme une des constantes du tempérament wallon ${ }^{3}$ ».

La spécificité liégeoise est complexe, le champ littéraire y étant caractérisé par un «mode de pensée que l'on a pu baptiser du nom de principautarisme ${ }^{4}$, nourri d'un positionnement d'indépendance, voire d'insularité à l'égard du reste de la Belgique francophone, et qui se voit renforcé par un «troisième facteur» qui «explique les contraintes qui pèsent sur la vie littéraire à Liège : la ville est un "centre secondaire" ou, pour renforcer l'oxymore, un "centre périphérique", qui se trouve pris dans une relation complexe avec les pôles d'attraction que sont Paris et Bruxelles ${ }^{5} »$. Liège subit comme toute périphérie l'attraction du pôle parisien, mais est soumis à la nécessité de composer avec le pôle bruxellois; pour Denis et Klinkenberg l'écrivain liégeois doit « frôler Bruxelles pour atteindre Paris ${ }^{6}$ ».

On verra plus loin comment ces facteurs, modernité, principautarisme et position de centre secondaire, ont pu intervenir dans la construction de l'objet qui nous occupe.

\section{LE PARCOURS D'IZOARD}

Il est utile de relever, dans le parcours de celui qui fera figure de « chef de file » du Groupe de Liège, les éléments qui ont pu contribuer à l'émergence du second et à la position occupée par le premier.

\footnotetext{
${ }^{1}$ Klinkenberg (Jean-Marie), Denis (Benoît), «Chapitre 12. Littérature : entre insularité et activisme », dans Le Tournant des années 70. Liège en effervescence, sous la direction de Jacques Dubois et Nancy Delhalle, avec la collaboration de Jean-Marie Klinkenberg, Bruxelles, Les Impressions nouvelles, 2010, pp. 237-253 ; Purnelle (Gérald), «La poésie à Liège dans les années 1980 : une transition “dialectique” », Art\&Fact, « Les années 1980 à Liège. Art et culture », n 31/2012, pp. 82-89.

${ }^{2}$ Voir Le Tournant des années 70. Liège en effervescence, cité en note 1.

${ }^{3}$ Klinkenberg et Denis, op. cit., p. 238.

${ }^{4}$ Ibid.

${ }^{5}$ Ibid., p. 239.

${ }^{6}$ Ibid., p. 240.
} 
Né en 1936, Izoard entre assez tôt dans deux revues locales de littérature (et non de poésie), Lettres 55 et L'Essai. En une dizaine d'années, sa trajectoire éditoriale connaît une expansion progressive, de Liège vers Bruxelles, puis Paris. Son institutionnalisation est relativement rapide ; Izoard paraît s'être rapidement tourné vers Bruxelles, en publiant en revues dès les années 60 et en intégrant les comités de plusieurs d'entre elles, dont Le Journal des poètes, auquel il collabore à partir de 1963 (il entre dans le comité de la revue et dans celui de la biennale internationale de poésie de Knokke). L'accès au champ français à travers les publications en revues date de la fin des années 1960.

Le parcours est comparable sur le plan éditorial : en dix ans, Izoard passe de l'édition locale - son premier recueil est publié à Liège par L'Essai, en 1962 - à Bruxelles, puis vient la consécration parisienne, avec trois recueils chez deux grand éditeurs: Voix vêtements saccages et La Patrie empaillée en 1971 et 1973 chez Grasset, Vêtu dévêtu libre chez Belfond en 1978, recueil qui lui vaut le Prix Mallarmé en 1979.

À Liège même, à partir de 1968, Izoard élit un lieu spécifique, la librairie Le Quai, pour y organiser des lectures, des rencontres et des entretiens.

En 1972, il réoriente radicalement le programme de la revue L'Essai, qu'il dirige alors, change son titre en Odradek et restreint son champ aux textes poétiques, en bannissant articles et critiques. Il se crée ainsi un instrument répondant à ses vœux et à ses besoins, et redéfinit l'offre liégeoise en matière de revues de poésie. Odradek comptera 17 livraisons de 1972 à 1979, sans se limiter à la publication de poètes liégeois ou même, plus largement, belges, mais en s'ouvrant à la France et à l'étranger. Dans un texte datable de septembre à décembre 1974, Izoard définit la revue :

«Odradek voudrait explorer le champ de l'écriture d'aujourd'hui, dans toute sa complexité, dans tous ses revers, dans toutes ses incohérences. [...] Les écrivants de Liège y ont leur place, et ceux d'ailleurs, en bons voisinages... / Ce que le poème profère dans ODRADEK, est-ce une réalité wallonne ? Certainement, et dans toute sa fragilité entêtée, dans toute sa ténacité de tête de houille...»

Ce programme est éloquent: s'inscrire dans son temps et, à travers un réseau en voie de constitution, affirmer une ambition internationale pour sortir d'un centrage purement local.

Enfin, sur le plan poétique, Izoard développe une poésie innovante, non classicisante, postsurréaliste, sensuelle et souvent hermétique. Dans cette veine, on peut dire qu'il fait école et que, rapidement, plusieurs cadets imitent son art de l'association des mots et de la fabrique des images.

\section{LE CHAMP POÉTIQUE LIÉGEOIS DANS LES ANNÉES 1970}

Au-delà du profil de son futur chef de file, quelques autres facteurs paraissent avoir mené à la constitution d'un « Groupe de Liège ».

Odradek n'est pas la seule revue liégeoise de poésie dans la première moitié des années 1970. La librairie Le Quai a sa propre revue, Les Cahiers de Roture, (1971-1972), et l'on peut citer en outre Donner à voir (Jean-Pierre Dobbels 1973-1975), ou Quetzalcoatl (1974-1975). Izoard participe à toutes ces revues, ce qui montre une volonté de fonder un réseau : tous les responsables de celles-ci seront intégrés dans la liste des membres du Groupe de Liège. 
Deux faits complètent cet état des lieux préalable à l'affirmation d'un Groupe de Liège. Fin 1972, Izoard découvre à la faveur d'un concours de jeunes poètes les textes d'Eugène Savitzkaya, alors âgé de 17 ans, et dont il défendra l'écriture avec constance. En 1972 toujours, l'artiste Robert Varlez, qui a lui aussi été « découvert» par Jacques Izoard dans les années 1960, fonde une maison d'édition artisanale baptisée l'Atelier de l'Agneau, qui se voue à la littérature contemporaine d'une certaine avant-garde, et à l'art.

Au moment de l'affirmation d'un Groupe de Liège en 1975, plusieurs conditions préalables sont donc réunies : depuis le début des années 1960, Izoard a atteint une position importante de poète, d'homme de réseau et d'animateur de la vie littéraire; il représente dans la poésie belge la tendance moderne et rénovatrice ; il fait figure de référence pour une génération plus jeune, qu'il cherche à fédérer autour de lui ; il dispose pour cela d'un lieu d'animation. Il est donc investi d'un important capital institutionnel, symbolique et relationnel.

1972 fut une année déterminante : trois événements ont pu cristalliser une volonté de donner une structure ou un nom à un réseau de plus en plus dense autour de Jacques Izoard: la transformation de L'Essai en Odradek; la création de l'Atelier de l'Agneau ; la découverte de Savitzkaya.

\section{5 : LE GROUPE DE LIÈGE}

Le Groupe de Liège a été officiellement « créé » ou révélé le 21 novembre 1975, dans la salle paroissiale de la commune de Grivegnée, dans la proche banlieue de Liège. Treize poètes furent présentés sous cette appellation par Francis Édeline, dont le discours introductif, intitulé «Le Groupe de Liège, aujourd'hui », mérite une analyse détaillée. N'étant pas intégré au groupe, Édeline paraît s'être vu confier une fonction de légitimation du groupe : il y déclare d'emblée saisir «l'occasion d'affirmer l'existence d'un Groupe de Liège, [....] parce qu'il est important de situer ce groupe aujourd'hui ». Édeline s'interroge d'abord sur la nature du groupe :

«S'agit-il d'un groupe au sens structuré et autoritaire du Surréalisme, ou de Tel Quel? Évidemment non. Il ne s'agit pas non plus, du moins pas exclusivement, du moutonnement émulatoire que l'on observe généralement dans le sillage d'une personnalité de premier plan. »

Procédant par la négative, sa réponse se fonde sur deux modèles qui lui permettent contrastivement de suggérer deux traits du Groupe de Liège : il n'est pas fondé sur l'autorité d'un seul, et ses bases ne sont pas dogmatiques. La seconde phrase n'affirme pas l'absence d'une personnalité de premier plan ; celle-ci existe, c'est Izoard, qui n'est pas nommé à ce stade, mais qu'Édeline présente plus tard dans ces termes : «J. Izoard fait figure de tête de file dans le groupe, même si sa modestie a toujours soigneusement respecté la personnalité des amis plus jeunes qui s'adressaient à lui. »

Les expressions employées — «personnalité de premier plan », Izoard « fait figure » de chef de file sans vraiment en adopter l'attitude et la posture — visent à suggérer un profil atypique caractérisé par une absence de hiérarchie, à travers les notions de non-structure, de non«moutonnement », de modestie du chef de file et de respect (mutuel).

Après avoir défini le groupe par la négative, Édeline poursuit par une analyse quasi sociologique : après avoir écarté une justification typologique et structurelle, il minimise le 
facteur géographique «d'une simple communauté d'habitat», au profit d'un argument plus relationnel, la « fraternité »:

«On pourrait assez justement parler selon moi d'une fraternité artisanale comme élément constitutif du Groupe de Liège. / Ce sont de jeunes écrivains qui aiment à se retrouver pour discuter des problèmes qui les préoccupent ou pour rencontrer ensemble des poètes étrangers de passage. Il est intéressant d'observer que les deux principaux foyers où ont lieu ces rencontres ne sont plus, comme naguère, de simples bistrots, mais les deux pôles de l'édition : l'atelier où s'élaborent les maquettes de livres [Édeline nomme ensuite l'Atelier de l'Agneau], et la librairie où on les vend [Le quai]. »

C'est donc bien, du moins dans le discours, la conjonction d'un lieu et d'un organe d'édition qui a pu déterminer le besoin apparemment collectif de se constituer et de s'affirmer en « groupe ». Les mots «fraternité artisanale» renvoient à la fois à cet idéal égalitaire déjà mentionné, et à cet ancrage dans la dimension matérielle et sociale. On ajoutera à ces facteurs la revue Odradek, Izoard précisant dans un texte datable de septembre à décembre 1974 que « les rencontres et animations du Quai ont été souvent nommées "soirées Odradek"... »

Les arguments d'Édeline se déplacent ensuite sur le plan de la poétique, jugeant que la légitimité du groupe doit passer par une unité minimale à cet égard :

«Mais cette définition externe, par la simple proximité de ses membres, n'est-elle, pas de ma part une dérobade devant la difficulté de saisir ce qui fait, de l'intérieur des œuvres, l'unité du Groupe de Liège ? J'aimerais hasarder quelques traits qui selon moi caractérisent au moins les courants principaux qui animent le Groupe. J'en retiendrai deux. »

Et l'orateur d'isoler «l'absence d'une recherche purement formaliste » («c'est le fond qui prime »), c'est-à-dire un rejet du formalisme jugé gratuit et propre aux avant-gardes françaises des années 70 - celui de revues telles que Tel Quel et peut-être Change (il existe l'un ou l'autre texte critique où Izoard s'en prend explicitement à ce formalisme au nom du sens et de la sensualité) — et, à travers « une sorte de sensualité généreuse », une thématique commune, un ancrage revendiqué dans une région — la Wallonie :

« Nos poètes sont sensibles au monde, aiment à manier sa pulpe, aiment à en jouir. Ils aiment en particulier la Meuse et ses Cités, ou les rudesses de l'Ardenne proche. Sans être régionalistes au sens désuet et un peu ridicule de ce mot, ils sont néanmoins d'une région et cette appartenance transparaît dans leurs poèmes. »

Si le Groupe de Liège, selon l'affirmation d'Édeline, se revendique comme lié à sa région et sans soumission esthétique à Paris, il s'agit, pour achever l'argumentation, de le situer dans une histoire proprement nationale ou locale de la poésie: le Groupe de Liège est «en évolution plutôt qu'en rupture », et la poésie liégeoise du moment s'alimenterait à trois influence : «le maniérisme baroque mais puissant de [Marcel] Thiry, la subtilité nuancée et intimiste d['Hubert] Dubois [et] «le sens généreux du concret de [Georges] Linze ».

L'argument n'est pas totalement convaincant sur le plan proprement poétique. S'il est vrai que le terme de « rupture » serait inadéquat et trop catégorique - le Groupe de Liège n'a rien d'une avant-garde prônant le rejet des prédécesseurs et la table rase -, le terme d' «évolution » est trop édulcoré : s'il y a bien, dans la poésie d'Izoard et de son groupe, un «maniérisme baroque » et un «sens généreux du concret», on ne peut guère les faire remonter directement à ces modèles; en outre, il y a dans la poésie d'Izoard, de ses 
contemporains et de ses épigones une réinvention de la poésie - même en demeurant dans l'orbe du lyrisme - qui tranche fortement par sa modernité et ses moyens rhétoriques sur le néo-classicisme.

Mais l'argument peut être pertinent, si l'on se fonde sur le parcours personnel d'Izoard. Il a pu, à ses débuts, solliciter les avis de Marcel Thiry; il a souvent rappelé les visites qu'il a faites au poète Hubert Dubois ; mais surtout il n'a cessé de fréquenter Georges Linze et de célébrer son œuvre et sa personnalité à travers plusieurs manifestations et textes critiques.

L'enjeu est toutefois bien plus institutionnel que purement poétique : non pas prolonger la poésie de Linze (très différente), mais poursuivre, précisément à travers des initiatives comme Odradek ou le Groupe de Liège, l'action du poète moderniste d'avant-guerre ; faire exister une vie poétique à Liège et lui donner une visibilité locale et externe.

Le discours d'Édeline pour expliquer le Groupe de Liège articule en deux temps sa dimension collective à « la personnalité de premier plan » qui l'anime et le mène. C'est en fin de séance, lors de la présentation de la «tête de file », qu'une dernière salve d'arguments à l'appui de l'image construite du groupe est lancée : un rassemblement de jeunes poètes attachés à la modernité et groupés autour d'une figure dont on ne nous dit pas explicitement qu'elle est charismatique, mais dans les qualités poétiques, humaines, sociales et «institutionnelles » sont soulignées : Édeline salue «l'inlassable animateur des rencontres, des confrontations, des revues » qui, « avec une discrétion admirable quant à son œuvre personnelle », « encourage et propulse ses jeunes amis »; pour conclure que «sans Jacques Izoard il y aurait peut-être des poètes liégeois, mais il n'y aurait pas de Groupe de Liège ». Édeline termine en soulignant à nouveau l'ancrage wallon des poètes groupés autour d'Izoard et se permet de «faire justice de ce préjugé peut-être dépassé d'une Cité Ardente [i.e. Liège] aux yeux perpétuellement tournés vers Paris »:

«L'Avant-Garde parisienne fut bien peu présente ce soir, et c'est tant mieux pour la personnalité du Groupe de Liège, et c'est tant pis pour l'idée toute faite de notre congénital lutétio-centrisme. »

En rejetant ce « lutétio-centrisme » liégeois explicitement dit « congénital », Édeline ne donne que la Wallonie comme cadre au Groupe de Liège. En fin de discours sont donc réaffirmés le principautarisme thématique du groupe et, partant, sa wallonitude. La prise de distance radicale à l'égard de Paris est fortement rhétorique. Par ce déni, autant que par le silence où est maintenue Bruxelles, elle affirme implicitement la vocation de Liège à compter comme centre sortant de sa position périphérique. Mais c'est aussi une prise de position à l'égard du champ littéraire francophone de Belgique : se revendiquer comme wallon, en cette période dialectique de l'histoire de nos lettres, revient à résoudre l'alternative des éthos centrifuge et centripète, singulièrement le second, propre au lundisme.

Au total, le discours d'Édeline dessine, voire stylise, la figure d'Izoard de façon à conférer au Groupe de Liège des traits flous mais simples et purement internes, tranchant sur le modèle archétypal du groupe littéraire. Souligner la personnalité d'Izoard tout en nuançant son autorité revient à appeler d'autres justifications de l'existence d'un groupe, dont sont essentiellement alléguées trois caractéristiques : le choix de la modernité en poésie, mais un rejet de l'avant-gardisme ; un attachement régionaliste à la Wallonie et à la ville de Liège, mais sur un mode censé contraster avec le passé ; une distance revendiquée à l'égard de Paris, sans que la position du centre secondaire par rapport à Bruxelles soit abordée frontalement. 
On notera que le Groupe de Liège n'affiche guère de discours programmatique ou théorique ; l'analyse unifiante d'Édeline est tirée des textes, en l'absence de tout manifeste un tant soit peu argumenté. Cet état de choses est bien en phase avec le portrait du chef de file, dont, par ailleurs, la poétique « est souvent imitée ».

\section{VIE ET SURVIE D'UN GROUPE}

Quels sont le fonctionnement et l'évolution du Groupe de Liège, après qu'il a été ainsi présenté en novembre 1975 ?

Durant la première moitié des années 70 s'est constitué autour de la personnalité de Jacques Izoard un réseau que Benoît Denis et Jean-Marie Klinkenberg ont pu nommer «la galaxie Izoard ». Composé de poètes, il est recruté pour une part parmi les animateurs de revues et de petites structures éditoriales présentes dans la région liégeoise. En fait aussi partie Robert Varlez, fondateur de l'Atelier de l'Agneau.

Ce réseau et sa personnalité centrale paraissent posséder leurs propres instruments de promotion, de diffusion et d'auto-affirmation. En 1975 on en compte trois : la revue Odradek, créée en 1972 par Izoard; l'Atelier de L'Agneau, né également en 1972; les rencontres Odradek de la libraire Le Quai ${ }^{7}$. On y ajoutera la création en 1977 de la revue mensuelle 25, fondée à partir de L'Atelier de l'Agneau par Robert Varlez avec l'aide d'Izoard. Le programme de cette revue, ouverte à la littérature actuelle, à la recherche et à l'expérimentation, aux arts graphies et à la critique, l'amène à prendre rapidement le pas sur Odradek, qui ne publie que des poèmes sans discours d'accompagnement, et en livraisons de volume restreint. Odradek cesse en 1979, mais on peut considérer que le mensuel 25 est rapidement devenu l'organe du réseau liégeois. Est-il aussi celui du Groupe de Liège ? Celuici survit-il à la proclamation de 1975 ?

Comparé aux contre-modèles évoqués par Édeline (le surréalisme et Tel Quel), le Groupe de Liège n'adopte guère les moyens conventionnels d'un groupe : les instruments du réseau ne se prêtent qu'à un usage très limité, voire inexistant, au service de la promotion nominale dudit Groupe de Liège. Le pragmatisme prend le pas sur la stratégie : la diffusion des textes des membres du groupe prime sur leur volonté d'intensifier l'affirmation de son existence.

\section{OÙ LE GROUPE EST-IL NOMMÉ ?}

Pour appréhender la destinée du groupe après la séance de 1975, nous disposons de cinq événements éditoriaux :

1. Dans son numéro d'avril 1978, le mensuel 25 publie une «Petite anthologie de la poésie française de Belgique », par laquelle Liège s'affirme dans le champ francophone de Belgique à travers une ouverture vers les autres sous-régions du champ poétique belge.

Ce choix des poètes ne cherche pas à être représentatif de la poésie belge contemporaine ; il repose uniquement sur un réseau, certes plus large que la seule région et le seul groupe

\footnotetext{
${ }^{7}$ « À l'époque, je prétendais avoir une attitude cohérente. Trois éléments faisaient partie de cette « cohérence »: la revue de poésie Odradek, les lectures publiques, au Quai, [...] et une petite maison d'édition L'Atelier de l'Agneau. Trois éléments complémentaires et qui se nourrissaient l'un l'autre. Le tout, bien entendu, avec la complicité de plusieurs amis » (Jacques Izoard dans un entretien avec Denys-Louis Colaux paru dans Orage Lagune Express, $\mathrm{n}^{\circ}$ 8, 1993).
} 
liégeois, mais on observe que les dix poètes habitant une commune de la province de Liège peuvent à peu près tous être intégrés au Groupe.

Le fascicule étant dépourvu de tout texte d'accompagnement, aucune mention n'est faite du Groupe de Liège ; en tant que réseau, il se manifeste par des actions éditoriales, non par un discours. La ville est toutefois affichée par l'image, à travers deux dessins de Robert Varlez : sur la couverture, Liège rayonne littéralement à travers la Belgique ; sur la première page, elle génère un arbre, métaphore visuelle de l'efflorescence poétique liégeoise revendiquée par le réseau groupé autour de son chef de file. La ville et sa région sont ainsi affirmées comme centre d'un champ.
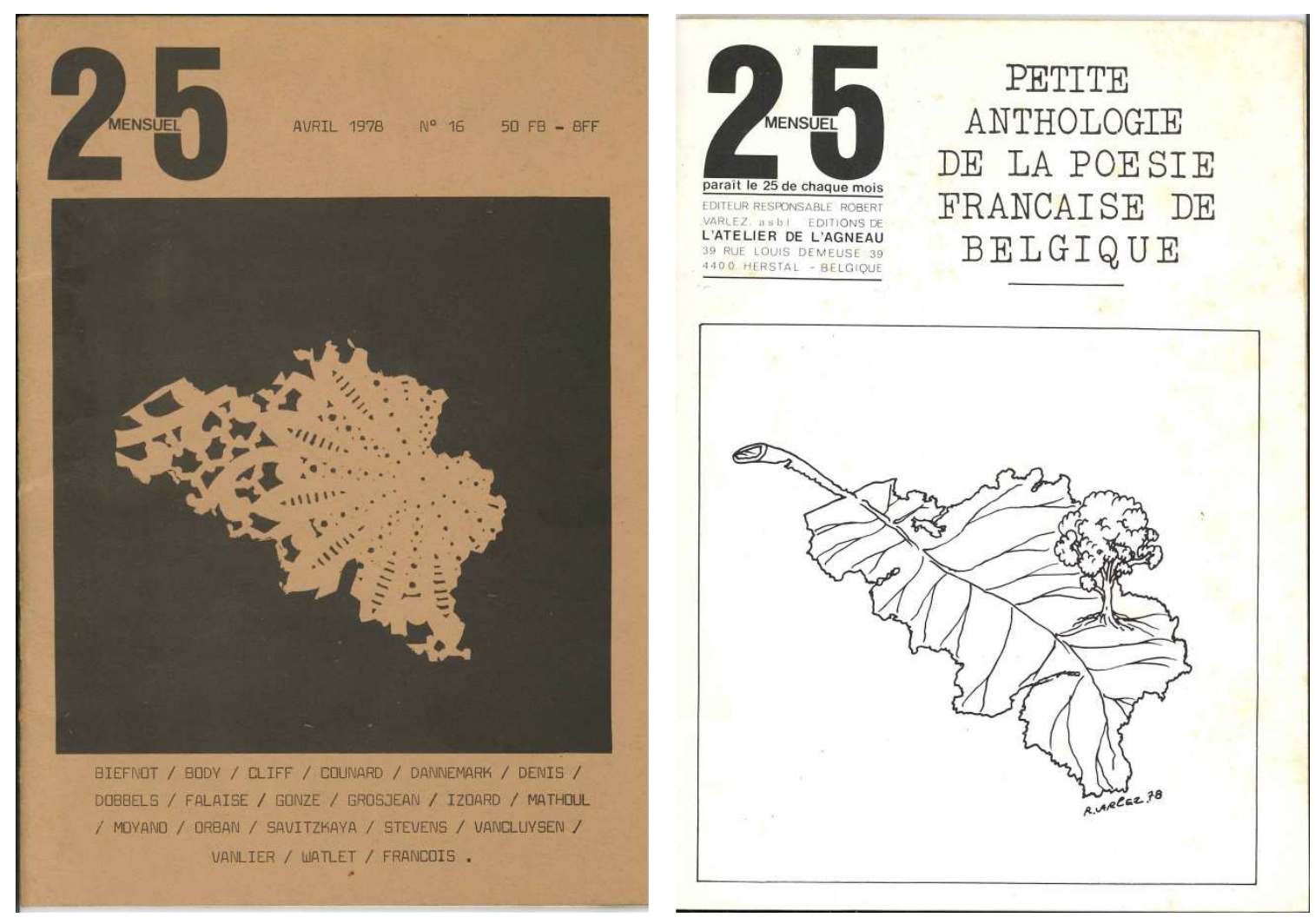

2. Deux moins plus tard, dans un numéro de la revue 25 consacré à son réseau de revues amies, Izoard, présentant sa revue dans un texte intitulé «Les cent poètes d'Odradek», mentionne le Groupe de Liège, en usant d'une rhétorique assez péremptoire : "La centaine de poètes publiés à ce jour dans Odradek forme une étrange galaxie. Le Groupe de Liège prétend impudemment en être le centre, le point vital, le noyau dur », ce qui paraît paraphraser à postériori les deux dessins de Varlez. Mais on notera que c'est le seul Jacques Izoard qui nomme le groupe, dans un texte personnel consacré à sa revue.

3. Le numéro d'octobre 1979 d'Odradek est consacré à «Six poètes de Liège ». Mais étrangement, il n'est fait mention nulle part du Groupe de Liège ; le seul lien que partagent les six poètes est rapporté à Odradek même, à ses rencontres, et non à l'existence d'un groupe. Par ailleurs leur nombre paraît trop faible pour être représentatif dudit groupe qui, en 1975, en comptait treize. Izoard évite ce qui pourtant était attendu : affirmer la fonction d'Odradek comme organe officiel du groupe. À cela on verra trois explications : ouvert vers l'extérieur, publiant régulièrement des poètes d'autres régions et d'autres pays, Odradek n'a pas la vocation de servir prioritairement la cause du groupe, même si certains poètes y ont été publiés ; cette fonction semble davantage assumée par le mensuel 25 , qui répugne pourtant à 
afficher la raison sociale du Groupe de Liège ; les jours de celui-ci en tant que concept et que réalité paraissent comptés...

4. L'Atelier de l'Agneau a publié en 1981 un gros volume intitulé Anthologie 80 et consacré à la modernité poétique de trois pays : la France, le Québec et la partie francophone de la Belgique. Une des trois préfaces au volume est due à Jacques Izoard et, consacrée à sa composante belge, elle a pour titre «L'autre autre pays, la poésie ». Le poète y parcourt le sommaire proprement belge de l'anthologie, sans grouper les poètes par sous-régions, mais en rappelant - pour la dernière fois, qu'il existe un Groupe de Liège :

«On trouvera dans cette anthologie plusieurs poètes qui ont fait ou qui font toujours partie de l'aire de Liège (Luc Bérimont utilisera l'expression «La nouvelle école de Liège »; le critique Francis Édeline parlera du «Groupe de Liège»). De 1970 à 1980, en effet, se produisirent à Liège de nombreux événements de prises de paroles, de mises en question de la poésie. Rejets. Refus. Ouvertures. Ruptures. Projets. Les cent manifestations du Quai, les cent poètes d'Odradek, les cent publications de l'Atelier de l'Agneau en constituent les témoignages irréfutables et tangibles. »

On relève que, si les manifestations et activités du groupe sont à nouveau fermement célébrées, le nom même du groupe est comme modalisé, et son existence même nuancée : "Luc Bérimont utilisera l'expression "La nouvelle école de Liège"; le critique Francis Édeline parlera du "Groupe de Liège". »

Certes, pour la première fois, le discours relatif au groupe allègue la caution d'une autorité externe au groupe, et même au champ proprement belge (c'est dans le Figaro-magazine du 24 mars 1979 que Bérimont nomme l'école), mais le nom du groupe est réduit au simple statut d'une expression. Du mot à la chose, le groupe en tant que réalité paraît avoir vécu ; et une école n'est pas un groupe.

Dans la suite, le propos d'Izoard se fait enfin polémique et, pour la première fois, Bruxelles est nommée comme destinataire antagoniste de cette activité et du discours qui l'accompagne :

«Tout cela n'alla pas sans susciter l'agacement de l'establishement littéraire, surtout bruxellois. Actuellement encore, plusieurs critiques officiels s'efforcent par tous les moyens de minimiser l'importance de ce mouvement, voire de l'occulter... »

On peut lire entre les lignes qu'il s'agissait bien de provoquer l'agacement de Bruxelles, mais aussi que cet effet fut peut-être plus désiré que produit, et en voie de disparition...

5. En novembre 1983 le mensuel 25 consacre un numéro aux écrivains de la province de Liège : « 50 écrivains de la province. Spécial Liège ». À nouveau, l'éditorial ne fait aucune mention du groupe, et tout y est sommairement, rapporté à la revue.

\section{COMPOSITION DU GROUPE}

Avant de conclure, il est intéressant de s'interroger sur la composition du Groupe de Liège dans la durée. Le tableau suivant juxtapose six listes : a) les poètes liégeois publiés par Izoard dans Odradek avant et après la soirée de Grivegnée de 1975 ; b) les 13 poètes de novembre 1975 ; c) ceux qui figurent dans la petite anthologie de 1978 ; d) les six poètes du numéro spécial d'Odradek; e) les Liégeois présents dans Anthologie 80 ; f) les poètes déjà apparus dans le tableau et repris dans le numéro «Spécial Liège » de 25 en 1983. 


\begin{tabular}{|c|c|c|c|c|c|c|c|}
\hline & $\begin{array}{c}\text { a. } \\
1972-1976\end{array}$ & $\begin{array}{c}\text { b. } \\
\text { nov. } 1975\end{array}$ & $\begin{array}{c}\text { c. } \\
1978\end{array}$ & $\begin{array}{c}\text { d. } \\
1979\end{array}$ & $\begin{array}{c}\text { e. } \\
1981\end{array}$ & $\begin{array}{c}\text { f. } \\
1983\end{array}$ & $\mathrm{Nb}$ \\
\hline Anne Body & 1972 & & & & & & 3 \\
\hline Michel Carpeau & & & & & & & 1 \\
\hline Roland Counard & 1973 & & & & & & 4 \\
\hline Jean-Pierre Dobbels & 1974 & & & & & & 4 \\
\hline Henri Falaise & 1974 & & & & & & 5 \\
\hline Jean-Marie Grosjean & 1975 & & & & & & 6 \\
\hline Gaspard Hons & 1974 & & & & & & 3 \\
\hline Christian Hubin & 1973 & & & & & & 2 \\
\hline Jacques Izoard & & & & & & & 4 \\
\hline Jean-Marie Mathoul & 1974 & & & & & & 6 \\
\hline Eugène Savitzkaya & 1973 & & & & & & 6 \\
\hline Daniel Simon & & & & & & & 1 \\
\hline Joseph Orban & & & & & & & 3 \\
\hline François Watlet & 1976 & & & & & & 4 \\
\hline Serge Czapla & & & & & & & 2 \\
\hline Jean-Claude Legros & 1974 & & & & & & 3 \\
\hline Alexandre Czapla & & & & & & & 2 \\
\hline
\end{tabular}

La seule liste explicitement liée au groupe est celle de 1975 ; y rapporter les autres relève pour une part de la conjecture, mais elle est fondée sur l'appartenance avérée des poètes au réseau d'Izoard et du mensuel 25.

Peut-on repérer le groupe dans ces publications, quand bien même il n'est plus nommé ? On constate une fluctuation assez importante de la composition :

- le groupe de 1975 est quasi exhaustivement puisé dans les poètes ayant publié dans Odradek: c'est donc bien le réseau d'Izoard qui fournit le groupe, dont il est clairement le centre ;

- quelques noms disparaissent rapidement, comme Gaspard Hons ou Christian Hubin, pour des raisons d'ordre poétique ou personnel ;

- un noyau dur, présent dès Odradek et jusqu'en 1983, est constitué des éditeurs et revuistes du début des années 1970, qu'Izoard a voulu fédérer autour de lui : Dobbels, Grosjean, Legros, Mathoul ; s'y ajoutent Savitzkaya et Falaise ; le Groupe de Liège est bien à l'origine une fédération fondée sur un réseau ;

- ce réseau se renouvelle par l'apport de nouveaux jeunes poètes : Joseph Orban ou François Watlet ;

- un sous-réseau familial occupe une place particulière : découvert en 1972 par Izoard, Savitzkaya est rejoint par ses trois frères, Jean-Pierre Dobbels, Serge et Alexandre Czapla. 


\section{CONCLUSION}

Il a bien existé un réseau de poètes autour d'Izoard, à qui il sert de base pour une stratégie dont le label «Groupe de Liège » est un élément rhétorique capital. Il profite d'un renouveau poétique propre aux années 70, touchant enfin Liège, pour développer une activité plus coordonnée. La motivation de cette démarche est double :

- personnellement, à la faveur d'une intégration affermie, mais isolée, dans l'institution belge et dans le champ français, sortir d'une position relativement solitaire et géographiquement éloignée (de Bruxelles et de Paris) ;

- plus collectivement, développer la visibilité et l'influence de Liège comme centre secondaire, en se fondant sur une ouverture à la fois large (la France) et orientée (la modernité).

Denis et Klinkenberg pointent qu'il y a là

« une sorte de grand écart: d'un côté un enracinement local, puissant et revendiqué, et de l'autre, simultanément, un désir d'ouverture très large, dès ses débuts [...]. Qu'entre les deux pulsions, il n'y ait pas d'intermédiaire (et qu'il n'y ait aucune aspiration à investir le champ bruxellois) est très significatif : c'est par là, bien plus encore que par ses thèmes et le son de sa voix, qu' Izoard est liégeois ${ }^{8}$. »

Mais les faits montrent qu'il s'agit pourtant bien de positionner Liège contre et face à Bruxelles - la préface d'Anthologie 80 et les illustrations de Varlez le montrent bien —, avec la France comme objectif stratégique, en tant qu'instance de légitimation, apte à fournir la dimension internationale nécessaire au groupe liégeois et à son leader, et donc un contrepoint à son régionalisme revendiqué.

L'invention du «Groupe de Liège » est donc ambiguë. Elle repose sur une réalité : un réseau d'éditeurs et de poètes, des organes de promotion, et répond au besoin stratégique d'une raison sociale, d'une étiquette; mais l'étiquette s'avère plus volatile que le réseau qu'elle prétend cristalliser. La création du Groupe de Liège relevant de l'initiative d'Izoard, il est significatif que lui seul emploie l'expression au-delà de la séance inaugurale de novembre 1975.

Le groupe n'a rien d'un ensemble structuré ; c'est d'ailleurs d'emblée revendiqué. Sa seule activité collective est la publication. Il n'a ni manifeste, ni discours théorique, ni dogme, ni stabilité de sa composition. Les organes soutiennent le réseau, mais n'usent guère du label du groupe.

Celui-ci apparaît comme une idée momentanée d'Izoard, datable de 1975 à 1978, après quoi le discours s'évapore. On ne trouve pas de trace d'une quelconque revendication d'appartenance de la part d'un des «membres » supposés du Groupe de Liège. Celui-ci survit néanmoins pendant quelques années sous la forme d'un noyau dur groupé autour d'Izoard et Savitzkaya.

Lancé à l'initiative d'un homme, le Groupe de Liège n'existe nominalement qu'autant que celui-ci continue à user de cette raison sociale. Il ne fut pas pour autant un simple mythe :

\footnotetext{
${ }^{8}$ Klinkenberg et Denis, op. cit., p. 245.
} 
«Groupe de Liège » est le nom momentanément et stratégiquement donné à un réseau qui, ne répondant guère à la définition du groupe littéraire, a même revendiqué cette spécificité. 